\title{
Endocrinological and metabolic effects of a polyherbal decoction of five Nigerian folkloric herbs on haloperidol induced hyperprolactinemia
}

\author{
Samuel, T. A. ${ }^{\star}$, Okonkwo, C. L., Ezeazuka, S. K. and Ekpoiba, A. J. \\ Department of Biochemistry, College of Medicine, University of Lagos, PMB 12003, Lagos, Nigeria.
}

Accepted 23 April, 2013

\begin{abstract}
Hyperprolactinemia is a major cause of infertility, and herbal remedies have been employed locally for treatment. This study was therefore designed to investigate the effects of a poly herbal mixture consisting of Bambusa vulgaris, Momordica charantia, Rauwolfia vomitoria, Ficus sur, and Clerodendrum capitatum on the reproductive hormones and metabolic parameters of haloperidolinduced hyperprolactinemic rats. Forty two female albino rats were divided into 7 groups of 6 in each group. Groups 2, 4, 5, 6 and 7 were given increasing doses $(2,3$ and $4 \mathrm{mg} / \mathrm{kg}$ body weight in five-daily increments) of haldol by intramuscular injection for 15 days after which they were treated for another 15 days with either $2.5 \mathrm{mg} / \mathrm{kg}$ body weight of bromocriptine (group 4 only) or 25,50 or $100 \mathrm{mg} / \mathrm{kg}$ body weight of the extract (groups 5, 6 and 7, respectively). Group 1 was given distilled water only while group 3 was given $25 \mathrm{mg} / \mathrm{kg}$ body weight extract only. After treatment, the animals were sacrificed and blood was taken from each group for plasma analysis of the reproductive hormones and metabolic parameters. Prolactin, follicle stimulating hormone, luteinizing hormone and estrogen were assayed. The fasting blood glucose, total protein and the lipid profile (total cholesterol and high-density lipoprotein (HDL) and low-density lipoprotein (LDL) cholesterol) were also determined. The result showed a dose-dependent significant reduction in the prolactin level by the extract with increase in the levels of the follicle stimulating hormone (FSH), lieutenizing hormone (LH) and estrogen. There was also a decrease in the levels of the triglycerides, LDL and total cholesterol while HDL was increased. In conclusion, the polyherbal preparation was able to reverse the hyperprolactinemia with its associated endocrine and metabolic changes in a manner comparable to that of bromocriptine.
\end{abstract}

Key words: Hyperprolactinemia, haldol, polyherbal decoction, reproductive hormones, metabolic indices.

\section{INTRODUCTION}

Hyperprolactinemia is a condition of elevated serum prolactin. Hyperprolactinemia may cause production and spontaneous flow of breast milk and disruptions in the normal menstrual period in women, and hypogonadism, infertility and erectile dysfunction in men (Nilsson et al.,
2009). Hyperprolactinemia can be a part of normal body changes during pregnancy and breastfeeding. It can also be caused by diseases affecting the hypothalamus and pituitary gland, disruption of the normal regulation of prolactin levels by drugs, medicinal herbs, heavy metals, 
and it may also be from disease of other organs such as the liver, kidneys, ovaries and thyroid (Mancini et al., 2008). The symptoms associated with hyperprolactinemia may be due to several factors like the direct effects of excess prolactin, such as the induction of galactorrhea or hypogonadism; the effects of the structural lesion causing the disorder (the pituitary tumor), causing conditions such as headaches, visual field defects, or external ophthalmoplegia. The symptoms may also be associated dysfunction of secretion of other anterior pituitary hormones (Asa, 2002). In some patients, however, it is not possible to elucidate the cause of hyperprolactinemia.

Use of prescription drugs is the most common cause of hyperprolactinaemia. Prolactin secretion in the pituitary is normally suppressed by the brain chemical dopamine. Drugs that block the effects of dopamine at the pituitary or deplete dopamine stores in the brain may cause the pituitary to secrete prolactin (Rosenbloom, 2010). These drugs include the major tranquillizers (phenothiazines), trifluoperazine (Stelazine), and haloperidol (Haldol) also an antipsychotic, antipsychotic medications in general; metoclopramide (Reglan), domperidone, cisapride used to treat gastro-oesophageal reflux and medication induced nausea (such as cancer drugs); and, less often, alpha-methyldopa and reserpine, used to control hypertension; and oestrogens and thyrotropin-releasing hormone (TRH) La (Torre and Falorni, 2007)

In Africa, much of the population relies on herbal treatment first, because of its affordability and the belief in the traditional healers and alternative therapists, before turning to conventional medicine, (Lee, 2003). A quarter of all new medicinal products registered around the world each year are derived from plants or other naturally occurring compounds. The biologically active defense compounds found within plants can also be useful for developing new drugs. As a result of their pharmacological or biological activity, medicinal plants can be used as a starting point for chemical and structural modification in order to improve the potency, selectivity and pharmacokinetics of drugs used in treating diseases (Mahdi et al., 2006).

Infertility is one of the major health problems in Nigeria in spite of the fast growing population. One of the major causes of infertility is hyperprolactinemia for which herbal treatment has been employed for treatment for a long time. The traditional healers lack the scientific understanding of hyperprolactinemia but they often diagnose it by one of its major symptoms, galactorrhea. One of the main herbal remedies for hyperprolactinemia is a decoction of five different local plants - Bambusa vulgaris, Momordica charantia, Rauwolfia vomitoria, Ficus sur, and Clerodendrum capitatum. In this research, we investigated the five herbs usually prescribed by the traditional healers to see if there was any scientific basis for the folkloric claims.

\section{METHODOLOGY}

\section{Collection and identification of plant materials}

The plant materials were purchased fresh from local market at Oyingbo, Lagos State Nigeria in July, 2012. They were identified and authenticated in the Botany Department of the University of Lagos, Akoka. The plants were given voucher numbers as listed in Table 1.

\section{Preparation of plant extract}

The extract of the herbs was obtained by leaves decoction. The leaves of the various plants were air dried and shredded. Seven liters of water was added to $2 \mathrm{~kg}$ of the dried shredded leaves (ratio $1: 1: 1: 1: 1)$ and this was boiled for one hour. The process was repeated twice by adding $4 \mathrm{~L}$ of water for $30 \mathrm{~min}$ to maximize the extraction. The total volume of extract obtained was $2.5 \mathrm{~L}$ which was concentrated to about $350 \mathrm{ml}$ by rotary evaporator and then freeze-dried.

\section{Experimental subjects}

Sixty female albino rats (Wistar strain) weighing between 110 and $135 \mathrm{~g}$ were purchased from Rattzmattaz Farms and Feeds Nig. Ltd. Satellite Town Lagos, Nigeria. The animals were housed in plastic cages with plastic bottom and wire mesh top with a $12 \mathrm{~h}$ light-dark cycle and adequate ventilation. The animals were allowed normal rat chow (Rattzmattaz Farms and Feeds Nig. Ltd. Satellite Town, Lagos) and water ad libitum throughout the study.

\section{Grouping and treatment of animals}

The female Wistar rats were divided into six treatment groups and a control group as shown:

1. GRP1: Distilled water;

2. GRP2: Haldol only;

3. GRP3: Low dose extract;

4. GRP4: Haldol + bromocriptine

5. GRP5: Haldol + low dose extract;

6. GRP6: Haldol + medium dose extract;

7. GRP7: Haldol + high dose extract.

Haldol (Janssen Pharmaceutical, Belgium) injection obtained from the pharmacy of the Federal Neuropsychiatric Hospital, Yaba, Lagos was administered to groups 2, 4, 5, 6 and 7 via intramuscular route for 15 days. The initial dose of the Haldol was $2 \mathrm{mg} / \mathrm{kg}$ body weight and was increased to 3 and $4 \mathrm{mg} / \mathrm{kg}$ bodyweight every 5 days. Groups 4, 5, 6 and 7 were afterwards treated for another 15 days with either $2.5 \mathrm{mg} / \mathrm{kg}$ body weight of bromocriptine or 25,50 or $100 \mathrm{mg} / \mathrm{kg}$ body weight of the extract. At the end of the treatment period, the rats were fasted overnight and were later anaesthetized with halothane. Blood samples were obtained by cardiac puncture into heparinized sample bottles.

\section{Biochemical assays}

The hormonal assays (prolactin, follicle stimulating hormone and luteinizing hormone) were determined by enzyme linked immunosorbent assay (ELISA) method using AccuBind ELISA kit (Monobind Inc. CA, USA). Total plasma protein and the lipid profile 
Table 1. Voucher numbers of plants.

\begin{tabular}{lc}
\hline Plant & Voucher number \\
\hline Ficus sur & LUH4850 \\
Momordica charantia & LUH 4853 \\
Rauwolfia vomitoria & LUH 4860 \\
Clerodendrum capitatum & LUH 4852 \\
Bambusa vulgaris & LUH 4856 \\
\hline
\end{tabular}

determination were done by enzymatic assay methods using analytical kits (RANDOX, United Kingdom). The glucose meter was evaluated using ACCU-CHEK glucose meter and test strips (Roche Diagnostics $\mathrm{GmbH}$, Germany).

\section{Statistical analysis}

Results are presented as mean \pm standard error of mean (SEM) for each group. Statistical analysis was done using the student's t-test to compare between the groups. A p-value less than 0.05 was considered statistically significant.

\section{RESULTS}

Table 2 shows the effects of administration of haldol and the polyherbal extract on the reproductive hormones. Administration of haldol significantly elevated the prolactin level $(p<0.05)$. For follicle stimulating hormone $(\mathrm{FSH})$ and lieutenizing hormone $(\mathrm{LH})$, the trends were similar as the lowest levels of the two hormones were found in the group treated with haldol only, and these values were significantly reduced $(p<0.05)$ compared to the controls. Administration of the herbal was seen to reduce the prolactin level and the high dose extract had the greatest effect $(p<0.05)$ of prolactin reduction which was close to the level observed in the group taking distilled water only. The FSH and $\mathrm{LH}$ levels too were found to rise, with the most significant effect $(p<0.05)$ found in the group given the highest dose of the extract. The pattern of the estrogen was also similar to that of $\mathrm{FSH}$ and $\mathrm{LH}$. Its lowest level was observed in the haldol only group and it increased in a dose-dependent manner.

The effects of haldol and the polyherbal preparation on plasma glucose and protein levels are shown in Table 3. There was a significant $(p<0.05)$ elevation of blood glucose with haldol administration. The extract was observed to significantly reduce the glucose level. The most significant effect was seen in the group with the highest dose of the extract. For the plasma protein, there were no significant changes across the various groups although there was some increase in the total protein in the haldol only group but it was not a significant one.

The effects of haldol and polyherbal preparation on the plasma lipid profile are shown in Table 4. There was a significant increase in the plasma triglycerides while there was a reduction in total cholesterol and $\mathrm{HDL}$ in the haldol only group. Administration of the decoction caused a dose-dependent reduction in the plasma triacylglycerol and LDL levels while causing significant $(p<0.05)$ increases in the levels of both total cholesterol and HDL.

\section{DISCUSSION}

Several herbal preparations have been employed by people from various races and tribes to treat hyperprolactinemia, and these preparations have been claimed to be effective (Hasani-Ranjbar et al., 2010). This research has investigated a polyherbal mixture which has been used in the southwestern part of Nigeria for a long time to treat hyperprolactinemia, with many success stories. Administration of haldol to the rats significantly raised the serum prolactin when compared to the group that took distilled water only. The polyherbal mixture even at the lowest dose was able to reverse the hyperprolactinemic effect of haldol. The prolactin lowering effect of the herbal mixture was found to be dose dependent, and at the highest dose of the extract, the serum prolactin was brought close to the level observed in the control group. The effect seen with the highest dose of this herbal preparation was close to that of bromocriptine which is a standard dopamine receptor agonist often used in the treatment of hyperprolactinemia.

Studies have shown that certain herbs truly possess anti-hyperprolactinemic effects. These herbal preparations include Vitexagnus cactus (Wuttke et al., 2003) and Peony-Glycyrrhiza Decoction (Yuan et al., 2008). Diterpenes found in these plants, and also as part of the constituents in our study polyherbal preparation, were reported to contribute to their prolactin lowering property (Shrivastava and Patel, 2007; Hasani-Ranjbar et al., 2010). There is dearth of data on the antihyperprolactinemic property of any of the plants in the polyherbal mixture studied in this work but this observed property might not be unconnected with the presence of the diterpenes.

We also found that the extract was able restore the level of gonadotropins, FSH and LH back to the levels in the controls. This was also in a dose dependent manner. The highest dose of the extract increased the levels of these hormones significantly and the levels were found to be close to the distilled water-only group. The extract might have increased the levels of these hormones by lowering the prolactin level. This is most likely to be the case since it is well established that high prolactin levels depresses the gonadotropins by its inhibitory effect on the $\mathrm{GnRH}$ which regulates the pituitary secretion of the gonadotropins (Nilsson et al., 2009 ). 
Table 2: Effect of administration of haldol and poly herbal preparation on the reproductive hormones of female Wistar rat.

\begin{tabular}{lcccc}
\hline Group/treatment & $\begin{array}{c}\text { Prolactin } \\
\text { (miu/ml) }\end{array}$ & $\begin{array}{c}\text { Follicle stimulating } \\
\text { hormone (miu/ml) }\end{array}$ & $\begin{array}{c}\text { Luteinizing } \\
\text { hormone }(\mathbf{m i u} / \mathbf{m l})\end{array}$ & Estradiol (pg/ml) \\
\hline GRP1 (Distilled water) & $62.67 \pm 4.79^{*}$ & $7.80 \pm 0.92^{*}$ & $5.20 \pm 0.45^{*}$ & $325.20 \pm 54.30^{*}$ \\
GRP2 (Haldol only) & $116.25 \pm 5.70$ & $2.10 \pm 0.37$ & $1.60 \pm 0.31$ & $104.00 \pm 48.54$ \\
GRP3 (Extract only) & $66.67 \pm 7.14^{*}$ & $6.40 \pm 0.92^{*}$ & $5.00 \pm 0.40^{*}$ & $360.67 \pm 58.95^{*}$ \\
GRP4 (Haldol + bromocriptine) & $60.60 \pm 2.70^{*}$ & $8.30 \pm 0.41^{*}$ & $6.50 \pm 0.77^{*}$ & $420.20 \pm 60.44^{*}$ \\
GRP5 (Haldol + low dose) & $94.00 \pm 4.36$ & $3.93 \pm 0.78$ & $4.40 \pm 0.26^{*}$ & $194.33 \pm 64.69$ \\
GRP6 (Haldol + medium dose) & $82.20 \pm 8.86^{*}$ & $6.75 \pm 0.54^{*}$ & $5.60 \pm 0.29^{*}$ & $276.40 \pm 50.25^{*}$ \\
GRP7 (Haldol + high dose) & $72.80 \pm 6.15^{*}$ & $8.10 \pm 0.79^{*}$ & $6.20 \pm 0.43^{*}$ & $432.40 \pm 60.44^{*}$ \\
\hline
\end{tabular}

Values are mean \pm SEM of six animals. *Significant difference when compared to Haldol only group $(p<0.05)$.

Table 3. Effect of administration of haldol and poly herbal preparation on glucose and total protein levels of female Wistar rats.

\begin{tabular}{lcc}
\hline Group/treatment & Glucose $(\mathbf{m g} / \mathbf{d l})$ & Total protein (mmol/L) \\
\hline GRP1 (Distilled water) & $106.00 \pm 4.27^{*}$ & $67.28 \pm 5.32$ \\
GRP2 (Haldol only) & $271.25 \pm 6.10$ & $72.97 \pm 5.53$ \\
GRP3 (Extract only) & $108.33 \pm 35.56^{*}$ & $65.82 \pm 5.30$ \\
GRP4 (Haldol + bromocriptine) & $95.00 \pm 42.12^{*}$ & $59.66 \pm 3.47$ \\
GRP5 (Haldol + low dose) & $109.00 \pm 47.13^{*}$ & $63.63 \pm 3.67$ \\
GRP6 (Haldol + medium dose) & $105.40 \pm 34.96^{*}$ & $61.03 \pm 3.52$ \\
GRP7 (Haldol + high dose) & $92.20 \pm 14.41^{*}$ & $67.15 \pm 3.43$ \\
\hline
\end{tabular}

Values are mean \pm SEM of six animals. *Significant difference when compared to Haldol only group $(p<0.05)$.

Table 4. Effect of administration of haldol and poly herbal preparation on lipid profiles of female Wistar rats.

\begin{tabular}{lcccc}
\hline Group/treatment & $\begin{array}{c}\text { Triglyceride } \\
\mathbf{~ m m o l / L}\end{array}$ & $\begin{array}{c}\text { Cholesterol } \\
\mathbf{m m o l} / \mathbf{L}\end{array}$ & $\begin{array}{c}\text { High density } \\
\text { lipoprotein } \\
\text { mmol/L }\end{array}$ & $\begin{array}{c}\text { Low density } \\
\text { lipoprotein } \\
\text { mmol/L }\end{array}$ \\
\hline GRP1 (Distilled water only) & $0.61 \pm 0.11^{*}$ & $3.08 \pm 0.03^{*}$ & $1.20 \pm 0.55^{*}$ & $0.73 \pm 0.35^{*}$ \\
GRP2 (Haldol only) & $1.73 \pm 0.03$ & $0.54 \pm 0.36$ & $0.85 \pm 0.11$ & $1.26 \pm 0.3$ \\
GRP3 (Extract only) & $0.63 \pm 0.17^{*}$ & $3.23 \pm 0.25^{*}$ & $1.37 \pm 0.06$ & $0.50 \pm 0.23^{*}$ \\
GRP4 (Haldol + bromocriptine) & $0.56 \pm 0.15^{*}$ & $3.34 \pm 0.26^{*}$ & $1.44 \pm 0.11^{*}$ & $0.10 \pm 0.15^{*}$ \\
GRP5 (Haldol + low dose) & $0.72 \pm 0.16^{*}$ & $2.68 \pm 0.15^{*}$ & $1.18 \pm 0.27^{*}$ & $0.61 \pm 0.44^{*}$ \\
GRP6 (Haldol + medium dose) & $0.68 \pm 0.14^{*}$ & $2.93 \pm 0.25^{*}$ & $1.41 \pm 0.89^{*}$ & $0.30 \pm 0.28^{*}$ \\
GRP7 (Haldol + high dose) & $0.58 \pm 0.05^{*}$ & $3.29 \pm 0.28^{*}$ & $1.56 \pm 0.10^{*}$ & $0.25 \pm 0.24^{*}$ \\
\hline
\end{tabular}

*Significant difference when compared to haldol only group $(p<0.05)$.

Similarly, from the results shown, the increase in the prolactin level strongly correlated with reduced gonadotropins and lowering of the prolactin also correlated with increase in the level of the gonadotropins. This strongly suggests that the extract effect was most likely through its ability to lower the prolactin level.
The effect of the polyherbal mixture was more striking on the serum estrogen levels. The extract was also able to increase the estrogen levels in a dose dependent manner. In fact, the highest dose had a very significant estrogen increasing effect when compared to the medium dose. The trend was similar to that observed for the 
gonadotropins but was greater in magnitude. Since the estrogens are part of the down line hormones of the hypothalamus-pituitary-gonadal axis, its level is regulated by the prolactin and the gonadotropins (Mohan and Marzher, 2010). However, the very significant effect seen might not be due to the gonadotropin stimulation only. Some components of the herbal mixture might have estrogen stimulation effect mediated through their constituents such as terpenoids (Telefo et al., 2004).

Many of the drugs that affect the reproductive hormones one way or the other have effect on the metabolic parameters, especially the energy metabolism, often leading to the problem of obesity/weight gain (Baptistaa et al., 1999). There was a significant elevation of blood glucose after the administration of haldol. The increase in blood glucose was likely to be due to hyperprolactinemia (Reis et al., 2004). The ability of bromocriptine to significantly bring down the glucose level was also major evidence that the high level of glucose observed in the haldol only group was most likely due to hyperprolactinemia. However, the possibility of haldol contributing to the increase in the blood glucose cannot be ruled out.

Haldol (or the typical antipsychotics) has been reported to raise the blood glucose (Wirshing et al., 2002). The plant was able to reverse the glucose concentration back to the level comparable to that of the control group. Though there was continuous reduction in the glucose levels as the extract dose increased, the difference was not so significant to conclude that the glucose lowering effect was dose dependent. Adeneye et al. (2008) have reported that $C$. capitatum which was one of the plants in this herbal mixture possess hypoglycemic property in Wistar rats. The extract of $M$. charantia has also been reported to stimulate cellular uptake of glucose (YibchokAnun et al., 2006).

Study has indicated that the primary constituents responsible for the hypoglycemic properties of $M$. charantia are charantin, insulin-like plant peptide, cucurbutanoids, momordicin, and oleanolic acids (Harinantenaina et al., 2006). Other constituent with hypoglycemic activity is $B$. vulgaris, as reported by Senthilkumar et al. (2011) which has the potency of reducing glucose significantly in hyperglycemia. The pattern of the plasma total protein did not show any significant difference across all groups, though there was a slight elevation in the plasma protein in the haldol only group. This indicates that both haldol and the extract do not seem to affect the plasma protein.

One of the commonest and most obvious effects of the typical antipsychotics is weight gain with increase in the mass of the adipose tissues (Huang and Lu, 2007). This correlates with the observation that there was a significant increase in the plasma triglycerides after haldol administration in this study. The herbal mixture reverted the effect on the triglycerides significantly and also in a dose dependent manner, and the triglyceride lowering effect with the highest dose was comparable to that observed for bromocriptine. This also suggests that the elevation of the triglyceride level might be secondary to hyperprolactinemia. The decoction also lowered plasma LDL level but increased HDL and total cholesterol levels. Chatuwedi (2005) confirmed that $M$. charantia reduces triglycerides (TG) and LDL but increases HDL. Adeneye et al. (2008) also reported that $C$. capitatum extract showed hypolipidemic effect in Wistar rats. The herbal mixture could therefore be said to have potential anti-obesity property and may prevent obesity related cardiovascular disorder.

There has been an increase in the trend of research using polyherbal preparations rather than the individual plants. This is mainly due to the fact that most herbal preparations used locally are often made from more than one herb. In this study, we sourced for the recipe which is locally reputed to be effective in the treatment of hyperprolactinemia and its symptoms. We found that the preparation made up of five different herbs was able to reverse the effects of haldol in rats. The results from this study support the folkloric claim of this polyherbal preparation. Some of the effects such as antihyperprolactinemic, anti-hyperglycemic and antihyperlipidemic effects of the herbal mixture in this study have been previously reported for some individual plants that made up the whole herbal preparation. However, more research should be carried out to be able to find out which particular plant is responsible for which effect and also to know which plant constituents are responsible. The effect seen in the polyherbal mixture might be a synergistic one which could only have been obtained in the mixed form. There is also the possibility that zeroing down on a particular plant or plant component might be more effective.

Further studies should be carried out to investigate the long term toxic effects of this polyherbal mixture. Some components of the herbs may have some toxic properties which may be of concern. There may be any herb-herb interaction which may be undesirable on the long term. Similarly, studies should also be carried out to investigate the drug-herb interactions that may possibly occur if the herbal mixture is taken alongside other synthetic drugs.

\section{REFERENCES}

Adeneye AA, Adeleke TI, Adeneye AK (2008) Hypoglycemic and hypolipidemic effects of the aqueous fresh leaves of Clerodendrum capitatum in Wistar rats. J. Ethnopharmacol. 116(1):7-10

Asa SL, Ezzat S (2002). The pathogenesis of pituitary tumours. Nature Rev. Cancer 2:836-849.

Baptistaa T, Reyesa D, Hernándeza L (1999). Antipsychotic Drugs and Reproductive Hormones: Relationship to Body Weight Regulation. Pharmacol. Biochem. Behav. 62(3)409-417. 
Harinantenaina L, Tanaka M, Takaoka S (2006). Momordica charantia constituents and antidiabetic screening of the isolated major compounds. Chem. Pharm. Bull. 54:1017-1021.

Hasani- Ranjbar S, Vahidi H, Taslimi S, Karimi N, Larijani B, Abdollahi $M(2010)$. A systematic review on the efficacy of herbal medicines in the management of human drug-induced hyperprolactinemia; potential sources for the development of novel drugs. Int. J. Pharmacol. 6:691-695.

Huang T, Lu C, Correlations between Weight Changes and Lipid Profile Changes in Schizophrenic Patients after Antipsychotics. Therapy. Chang Gung Med. J. 30:26-32.

La Torre D, Falorni A (2007). Pharmacological causes of hyperprolactinemia. Clin. Risk Manag. 3(5):929-951.

Lee S, Ahn J, Kim S, Kim J, Han S, Jung M, Chung I (2003). Variation in isoflavone of soybean cultivars with location and storage duration. J. Agric. Food Chem. 51:3382-3389.

Mahdi JG, Mahdi AJ, Mahdi AJ, Bowen ID (2006). The historical analysis of aspirin discovery, its relation to the willow tree and its antiproliferative and anti-cancer potential. Cell Prolif. 39:147-155

Mancini T, Casanueva FF, Giustina A (2008). Hyperprolactinemia and Prolactinomas. Endocrinol. Metab. Clin. North Am. 37(1):67.

Mohan K, Marzher S (2010). FSH, LH, Prolactin levels in infertile women in North Chennai, Tamilnadu. J. Biosci. Res. 1(4):279-284

Nilsson LA, Roepstorff C, Kiens B (2009). Prolactin suppresses malonyl-CoA concentration in human adipose tissue. Horm. Metab. Res. 41(10):747-751.

Reis FM, Santos MA, Reis AM, Coimbra CC (1994). Effects of hyperprolactinemia on plasma glucose and prolactin in rats exposed to ether stress. Physiol. Behav. 56(3):495-499.
Rosenbloom AL (2010). Hyperprolactinemia with Antipsychotic Drugs in Children and Adolescents Int. J. Pediatr. Endocrinol. (159402)1-6

Senthilkumar MK, Sivakumar P, Faisal C, Rajesh V, Perumal P (2011). Evaluation of anti-diabetic activity of Bambusa vulgaris leaves in streptozotocin induced diabetic rats. Int. J. Pharm. Sci. Drug Res. 3(3):208-210.

Shrivastava N, Patel T (2007). Clerodendrum and Heathcare: An Overview. Med. Aromat. Plant Sci. Biotechnol. 1(1):142-150.

Telefo PB, Moundipa PF, Tchouanguep FM, (2004). Inductive effect of the leaf mixtures extracts of Aloe buettner, Justcia insularis, Dicliptera verticillata and Hibiscus macranthus on the invitro production of estradiol. J. Ethnopharmacol. 91(2-3):225-30.

Yibchok-Anun S, Adisakwattana S, Yao CY (2006). Slow acting protein extract from fruit pulp of Momordica charantia with insulin secretagogue and insulinomimetic activities and insulinomimetic activities. Biol. Pharm. Bull. 29:1126-1131.

Yuan HN, Wang CY, Sze CW, Tong Y, Tan QR, Feng XJ, Liu RM, Zhang JZ, Zhang YB, Zhang ZJ (2008). A randomized, crossover comparison of herbal medicine and bromocriptine against risperidone-induced hyperprolactinemia in patients schizophrenia. J. Clin. Psychopharmacol. 28(3)264-370. 